\title{
Análisis multivariado de la morfometría y merística del robalo Eleginops maclovinus (Cuvier, 1830)
}

\author{
Morphometric and meristic multivariate analysis in patagonian blennie \\ Eleginops maclovinus (Cuvier, 1830)
}

\author{
Santiago Gacitúa ${ }^{1,2}$, Ciro Oyarzún ${ }^{1}$ y Rodrigo Veas ${ }^{1,3}$ \\ ${ }^{\prime}$ Departamento de Oceanografía, Universidad de Concepción, Casilla 16O-C, Concepción, Chile \\ ${ }^{2}$ Programa de Magíster en Ciencias Mención Pesquerias, Universidad de Concepción, Concepción, Chile \\ ${ }^{3}$ Programa de Doctorado en Oceanografia, Universidad de Concepción, Concepción, Chile \\ sgacituab@entelchile.net
}

\begin{abstract}
The Patagonian blennie is one of the few Notothenioidei distributed outside Antarctic or subantarctic waters. It is a coastal species generally associated with estuarine systems and has been exploited as a fishery resource in Chile from 1957. In this research we studied the variability in morphometric and meristic characters of E. maclovinus caught by fishermen at the Bío Bío river mouth $\left(36^{\circ} 48^{\prime} 30^{\prime \prime} \mathrm{S}\right.$; $\left.73^{\circ} 10^{\prime} 00^{\prime \prime} \mathrm{W}\right)$ and Puerto Montt (41 $\left.{ }^{\circ} 29^{\prime} \mathrm{S} ; 7^{\circ}{ }^{\circ} 7^{\prime} \mathrm{W}\right)$. From the net truss design on fish morphology, 27 morphometric characters emerged, also, the information of 8 meristic characters was registered. All the information was analyzed through multivariate approximations. Morphometric variables and meristic counts that explain some differentiation between localities through Principal Components Analysis (PCA) were determined. The groups and individuals were compared using Multiple Discriminant Analysis (MDA) in order to determine the reclassification percentages according with the fish shape. Using Covariance Analysis (ANCOVA) we tested if size was a variable that influenced the morphometric results. Our results revealed differences in morphometry between the individuals coming from the two localities but those differences were not completely independent of body size. The results for meristics counts did not allow recognition of meaningful differences between localities. The morphometrics results showed that the length, as descriptor of body size, would stress the differences between populations.
\end{abstract}

Key words: Patagonian blennie, ACP, DA, ANCOVA

\section{Introducción}

El robalo, Eleginops maclovinus (Cuvier, 1830), taxón monotípico en la familia Eleginopidae (Eastman \& Eakin 2000), es una de las pocas especies del Suborden Notothenioidei que no se encuentra restringida por la Convergencia Antártica al Océano Polar, ya que su distribución geográfica abarca por el Océano Atlántico desde la desembocadura del río de la Plata hasta el extremo sur de Sudamérica, incluyendo las islas Falkland,
Resumen.- El robalo es uno de los pocos Notothenioidei que está distribuido fuera de aguas antárticas o subantárticas. Es una especie costera asociada generalmente a sistemas estuarinos y se ha explotado en Chile como recurso pesquero desde 1957. El presente trabajo analizó la posible variabilidad en los caracteres morfométricos y merísticos de E. maclovinus, para ejemplares provenientes de la pesquería de la desembocadura del río Bío Bío (36 48'30"S; $\left.73^{\circ} 10^{\prime} 00^{\prime \prime} \mathrm{W}\right)$ y Puerto Montt (41 ${ }^{\circ} 29^{\prime} \mathrm{S}$; $\left.72^{\circ} 57^{\prime} \mathrm{W}\right)$. Del diseño de entramado sobre la morfología de los peces surgieron 27 caracteres morfométricos, y además, se registró la información de 8 caracteres merísticos, los que fueron analizados con una aproximación multivariada. Mediante Análisis de Componentes Principales (ACP), se determinaron las variables morfométricas y recuentos merísticos, que explican una mayor diferenciación entre localidades. Utilizando Análisis Discriminante Múltiple (ADM) se compararon además los grupos e individuos para determinar los porcentajes de reclasificación de acuerdo a la forma de los peces. Utilizando Análisis de Covarianza (ANCOVA) se estableció si la talla era una variable que influía en los resultados morfométricos obtenidos. Los resultados revelaron la existencia de diferencias en la morfometría entre los individuos provenientes de ambas localidades, pero dichos resultados no fueron completamente independientes del tamaño de los peces. Los resultados para los recuentos merísticos no permitieron reconocer diferencias significativas entre localidades para ninguno de los recuentos. De los resultados morfométricos se estableció que la talla como descriptor del tamaño, acentuaría las diferencias encontradas entre las poblaciones.

Palabras clave: Notothenioidei, ACP, ADM, ANCOVA

y desde este extremo hasta Valparaíso por el Océano Pacifico (Miranda 1967, Guzmán \& Campodónico 1973, Zama \& Cárdenas 1984a, Pequeño et al. 1995). E. maclovinus sería una forma moderna posiblemente derivada de un grupo antiguo de la familia Nototheniidae, el cual se dispersó desde algún punto al sur de la zona austral de América (Pequeño 1989), o por el contrario, la alternativa más aceptada es que representaría la especie más plesiomórfica (Eastman \& McCune 2000, Iwami 2004), hermana del resto de las Notothenioidei modernas 
y que aparentemente se habría aislado luego de la apertura del paso Drake y la formación de la Zona Frontal Polar, lo cual habría ocurrido hace unos 25 a 22 millones de años (Balushkin 1992, Klingenberg \& Ekau 1996, Lecointre et al. 1997, Bargelloni et al. 2000a, Bargelloni et al. 2000b). Recientemente, Near (2004) estimó que $E$. maclovinus se habría separado hace aproximadamente unos 38 millones de años a partir de su posible ancestro Proeleginops grandeastmanorum.

E. maclovinus es reconocida como una especie netamente costera, con rasgos nerítico-demersal a bentonerítico, encontrándose normalmente asociada a zonas estuarinas (Pequeño 1979). Durante una etapa de su vida entra desde el mar a la desembocadura de ríos, donde permanece viviendo en las cercanías del fondo, alimentándose de algas y pequeños invertebrados (Guzmán \& Campodónico 1973, Pequeño 1987, Martin \& Bastida 2008), presenta diferencias dietarías a lo largo de su distribución geográfica debido a la variedad de oferta ambiental entre una zona y otra (Licandeo et al. 2006), depredando incluso sobre juveniles de salmónidos (Zama \& Cárdenas 1984b).

Licandeo et al. (2006) revisaron la edad y crecimiento en la zona-centro sur de Chile encontrando resultados similares a Panozo (1996) para la talla de reversión sexual, con un rango entre $36-40 \mathrm{~cm}$ LT, pero menores a los 53 $\mathrm{cm}$ informados por Brickle et al. (2005) para las Islas Falkland, en donde se confirmó el hermafroditismo protándrico reportado por otros autores (Calvo et al. 1992, Panozo 1996), y que E. maclovinus posee una estrategia reproductiva muy diferente de otros nototenioideos que se desarrollaron en ambientes antárticos y subantárticos, pareciendo tener, una historia evolutiva diferente y con rasgos como hermafroditismo protándrico, huevos pequeños y alta fecundidad, que son más característicos de peces de latitudes templadas (Brickle et al. 2005). Para robalos provenientes de las I. Falkland, Estrecho de Magallanes y Punta Arenas, Mazzei et al. (2008), establecieron que el cariotipo de E. maclovinus está compuesto por 48 cromosomas, similar a la mayoría de los perciformes, no presentando variabilidad intraespecífica entre las localidades muestreadas.

El robalo, como especie recurso, ha estado presente desde los primeros asentamientos poblacionales en la costa de Chile (Torres et al. 2007), y ha sido un componente habitual en las estadísticas nacionales de los últimos 50 años (SAG 1976).

Desde el punto de vista poblacional, sin embargo, sólo el trabajo de Gómez (1996) pretende establecer diferencias desde una perspectiva genética para $E$. maclovinus, encontrando muy baja variabilidad genética para muestras provenientes del río Bío Bío y Calbuco. El autor encuentra un sólo locus polimórfico de 19 analizados, concluyendo que el robalo es una especie muy poco variable genéticamente.

Por otro lado, siendo una especie con un rango de distribución tan amplio y que debe enfrentar una gran variedad de aguas, desde zonas con marcadas surgencias costeras hasta aguas subantárticas, sería esperable una mayor variedad de respuestas fenotípicas, y en consecuencia presentar tipos morfológicos propios de cada zona.

El análisis de la morfometría y merística, como técnica ha sido ampliamente utilizada con resultados satisfactorios principalmente en la determinación de procesos microevolutivos en varías especies de importancia comercial (George-Nascimento \& Arancibia 1992, Cortés et al. 1996, Oyarzún 1997, Hernández et al. 1998). Esta aproximación se basa en la variación morfológica de las distancias medidas a partir de un entramado entre una serie de hitos corporales, y del recuento de los elementos que componen las estructuras a lo largo, o en partes específicas del cuerpo del pez (Humphries et al. 1981, Strauss \& Bookstein 1982, Bookstein et al. 1985, Winans 1987, Cadrin 2000, Fitzgerald et al. 2002). Teóricamente esta caracterización sistemática de la geometría de la forma del pez, aumenta la posibilidad de extraer diferencias morfométricas con un significado biológico dentro y entre especies (Winans 1987, Fitzgerald et al. 2002).

El objetivo del presente trabajo consiste en comparar la posible variación en los caracteres morfológicos y merísticos de E. maclovinus para las áreas central y sur de Chile.

\section{Material y métodos}

\section{Obtención de las muestras}

Entre febrero y noviembre de 1996 se recolectaron 96 ejemplares de E. maclovinus provenientes de los desembarques de la pesquería artesanal, desde la desembocadura del río Bío Bío $(\mathrm{n}=58)$ y 38 ejemplares de la localidad de Puerto Montt (Fig. 1). Para cada ejemplar se determinó su longitud total en centímetros, utilizando un ictíometro $( \pm 0,1 \mathrm{~cm})$.

Para la obtención de las medidas morfométricas se consideraron 12 hitos morfológicos (Fig. 2a), de los que se obtuvo una red de 27 distancias corporales (Fig. 2b), siguiendo lo descrito por Humphries et al. (1981), Strauss \& Bookstein (1982), Bookstein et al. (1985) y Winans (1987), e incorporando una variable que expresa el ancho del pez medido entre las inserciones de la aleta pectoral 


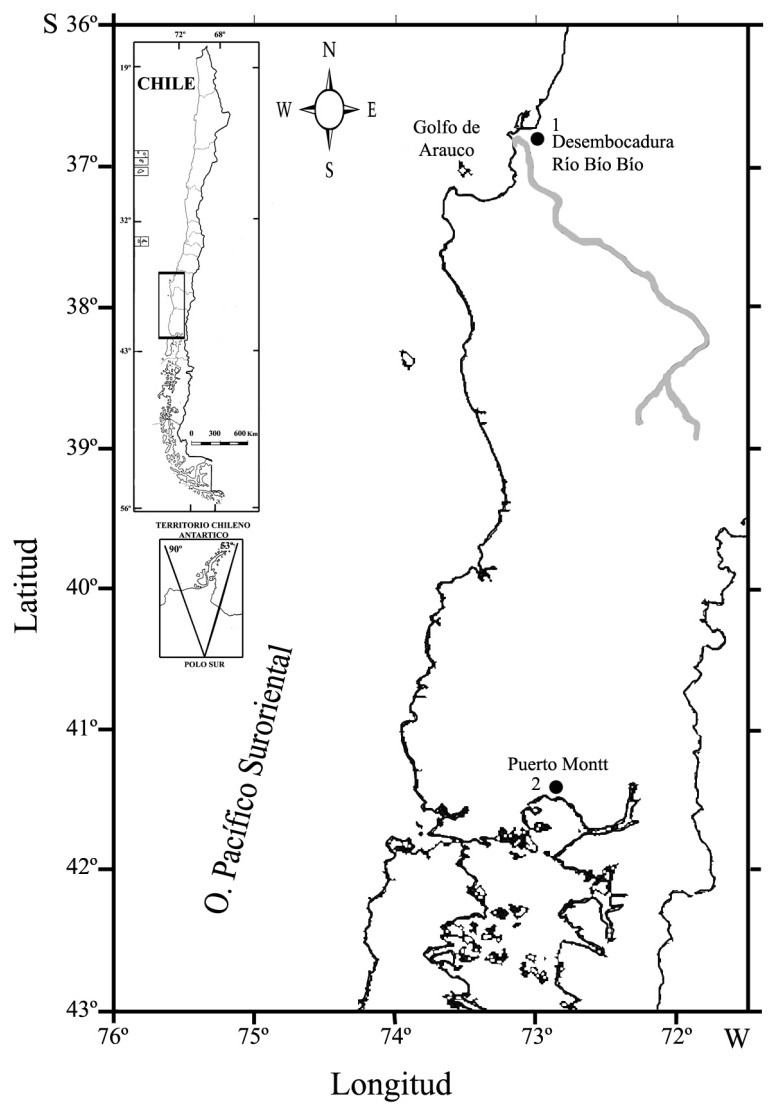

Figura 1

Zona de estudio mostrando las dos localidades de muestreo (1= río Bío Bío; 2= Puerto Montt)

Study zone showing the sampling localities ( $1=$ Bío Bío river; $2=$ Puerto Montt)

sobre el hito 12 de la red morfométrica (Fig. 2). Las longitudes fueron obtenidas utilizando un pie de metro $( \pm 0,01 \mathrm{~mm})$, y en ciertas ocasiones un compás de dos puntas (leído en una regla graduada $\pm 0,01 \mathrm{~mm}$ ).

Para la obtención de los datos merísticos, en cada pez se registró el recuento de las siguientes estructuras: 1. Espinas en la primera aleta dorsal (RD-I); 2. Radios en la segunda aleta dorsal (RD-II); 3. Radios en la aleta anal (RA); 4. Radios en la aleta pectoral (RP); 5. Vértebras precaudales (VPREC); 6. Vértebras caudales (VC); 7. Branquiespinas superiores en el primer arco branquial (BSUP), y 8. Branquiespinas inferiores en el primer arco branquial (BINF).

\section{Morfometría y merística}

Dado que las medidas morfométricas constituyen datos continuos (Humphries et al. 1981, Strauss \& Bookstein
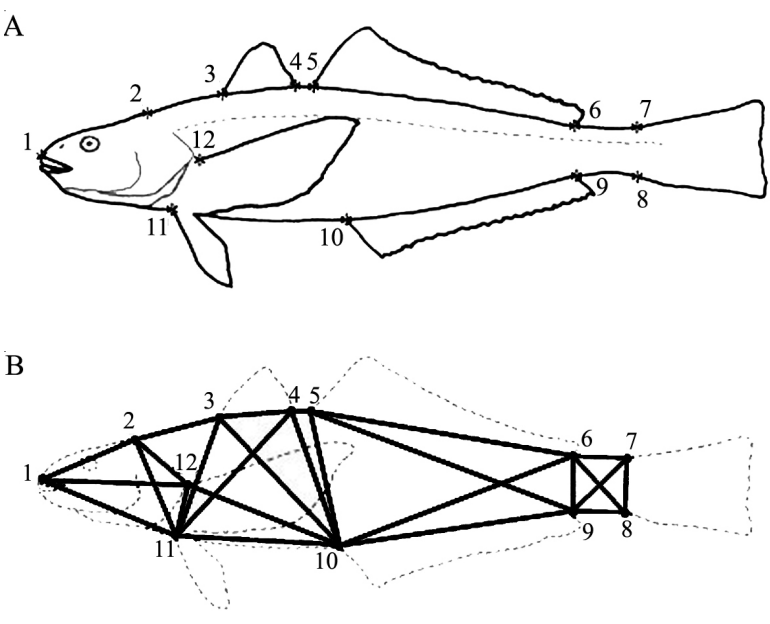

Figura 2

a) Distribución de los principales hitos morfológicos considerados para la construcción de la red de medidas morfométricas, b) Red de tramado de las diferentes medidas morfométricas registradas

a) Distribution of main morphological landmarks considered for the net truss construction, b) net truss of recorded morphometric measures

1982, Winans 1987), estos datos fueron transformados al logaritmo base 10 para evitar las correlaciones existentes entre las varianzas y medias de las mediciones (Sokal \& Rohlf 1979).

Los caracteres merísticos, por su naturaleza discreta, fueron transformados a la raíz cuadrada de la medida, más 0,5 , debido a que la varianza y la media son iguales, por lo que no son independientes, sino que varían idénticamente (Sokal \& Rohlf 1979).

\section{Análisis estadístico}

Para el análisis de los datos se utilizó a los individuos en su conjunto sin realizar una separación de las muestras por sexos, aún cuando pudieran existir diferencias en la morfometría de machos y hembras en una misma localidad o entre localidades. Lo anterior se debió a que, como se ha informado por Calvo et al. (1992), Panozo (1996) y Brickle et al. (2005), E. maclovinus es una especie hermafrodita secuencial protándrica, por lo que una inspección visual de las gónadas no permite establecer con total seguridad el sexo de un individuo determinado.

Previo a los análisis estadísticos multivariados se procedió a evaluar la normalidad de los datos, utilizando la prueba W de Shapiro \& WiIk (Shapiro et al. 1968), para cada una de las 27 variables morfométricas. 
La determinación de los trazos morfométricos a ser empleados en los posteriores análisis multivariados, fue realizada por medio de un análisis de conglomerados sobre una matriz de correlación (1-Pearson, como medida de distancia), seleccionando aquellas variables de menor correlación, y en aquellas combinaciones donde se presentó un valor alto de correlación se excluyó aquella variable que tuviera menor normalidad (prueba $\mathrm{W}$ de Shapiro \& Wilk). La selección de una matriz reducida de variables permite realizar un análisis que puede encontrar diferencias significativas donde realmente existen (Misra \& Easton 1999).

Una vez seleccionadas las variables, se realizó un Análisis de Componentes Principales (ACP) de los datos morfométricos y merísticos, sobre una matriz de covarianza, para la separación entre localidades. Además, se utilizó un Análisis Discriminante Múltiple (ADM) sobre las variables seleccionadas, y la posterior aplicación de una prueba $\mathrm{F}$ univariada para cada una de ellas, usando como variable dependiente las medidas morfométricas y merísticas, y como fuente de variación la localidad. Para determinar los posibles efectos de la localidad y la talla de los peces, se realizaron Análisis de Covarianza (ANCOVA) tomando como variable dependiente los puntajes del primer y segundo eje de los ACP realizados, como fuente de variación la localidad y como covariable para ambas la longitud total (LT).

Además, debido a que las muestras provenientes del río Bío Bío y Puerto Montt presentaban diferentes rangos para la distribución de frecuencias de talla, y con el objeto de explorar el posible efecto de la talla, se realizó un segundo análisis considerando sólo aquellos ejemplares que se encontraban en un rango de tamaño similares, entre 42 y $52 \mathrm{~cm}$, de longitud total (Fig. 2). De esta forma la submuestra quedó compuesta por 57 individuos de los cuales 20 correspondieron a la desembocadura del río Bío Bío y 37 a Puerto Montt, siendo los datos sometidos a los mismos procedimientos analíticos ya descritos.

Todos los análisis fueron realizados con los programas estadísticos SYSTAT 8.0 (SPSS Inc. 1998) y STATISTICA 6.0 (StatSoft Inc. 1998).

\section{Resultados}

La distribución de frecuencias de tamaños se muestra en la Fig. 3. Luego de la prueba de normalidad y de los análisis de correlación y de conglomerados, la matriz final de datos morfométricos se redujo de 27 a 17 variables; todos los análisis y discusión posterior están referidos a ese conjunto de variables (Tabla 1).

El ACP (Tabla 1), muestra que las dos primeras

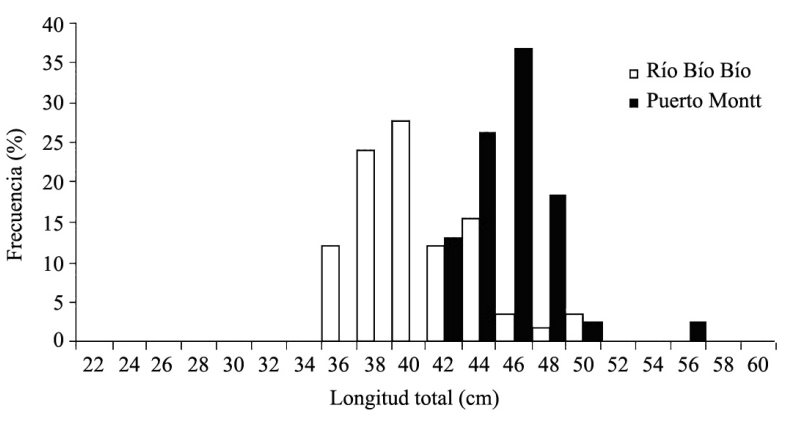

Figura 3

Distribución de frecuencias de talla (\%) para E. maclovinus en cada localidad (río Bío Bío $\mathbf{n}=58$; Puerto Montt $\mathbf{n = 3 8}$ )

Length frequency distribution (\%) of E. maclovinus for each locality (Bío Bío river $n=58$; Puerto Montt n=38)

Tabla 1

Componentes de carga de las 17 variables morfométricas con las tres primeras componentes principales para $E$. maclovinus $(*=$ mayor varianza explicada por variable para cada componente; $\mathbf{C P i}=\mathrm{i}$-ésima componente principal; V.E.= varianza explicada)

Component loadings of 17 morphometric variables for three first principal components in E. maclovinus ( $^{*}=$ highest variance per variable for each component; $\mathrm{CPi}=\mathrm{i}$-th principal component; V.E.= explained variance)

\begin{tabular}{lccr}
\hline Trazo & CP1 & CP2 & CP3 \\
\hline $1-2$ & $0,023^{*}$ & 0,004 & 0,004 \\
$1-12$ & $0,035^{*}$ & 0,001 & 0,009 \\
$2-3$ & $0,040^{*}$ & 0,003 & 0,004 \\
$2-12$ & $0,035^{*}$ & 0,006 & 0,005 \\
$3-10$ & $0,046^{*}$ & 0,009 & $-0,002$ \\
$3-11$ & $0,033^{*}$ & 0,007 & $-0,015$ \\
$5-6$ & $0,042^{*}$ & 0,003 & 0,006 \\
$5-9$ & $0,044^{*}$ & 0,004 & $-0,000$ \\
$5-10$ & $0,044^{*}$ & 0,009 & $-0,008$ \\
$6-9$ & $0,030^{*}$ & 0,005 & $-0,002$ \\
$6-10$ & $0,039^{*}$ & 0,007 & 0,008 \\
$7-8$ & $0,029^{*}$ & $-0,006$ & 0,008 \\
$7-9$ & $0,037^{*}$ & $-0,006$ & 0,016 \\
$8-9$ & 0,037 & $-0,065^{*}$ & $-0,005$ \\
$10-12$ & $0,046^{*}$ & 0,004 & $-0,012$ \\
$11-12$ & $0,040^{*}$ & 0,005 & $-0,009$ \\
$12-12 b$ & $0,028^{*}$ & 0,006 & 0,001 \\
& & & \\
V.E. (\%) & 66,498 & 12,969 & 3,053 \\
V.E. (\% acum.) & 66,498 & 79,467 & 82,520 \\
\hline
\end{tabular}

componentes principales explican $79,5 \%$ de la varianza total, siendo la primera componente $(66,5 \%$ de la varianza) la que más aporta a la separación entre localidades (Fig. 4), ya que todas las medidas covariaron mayormente con dicha Componente Principal (CP1), excepto la variable 8-9 que lo hizo con la segunda (Tabla 1). El resultado del ANCOVA (Tabla 2) realizado sobre 


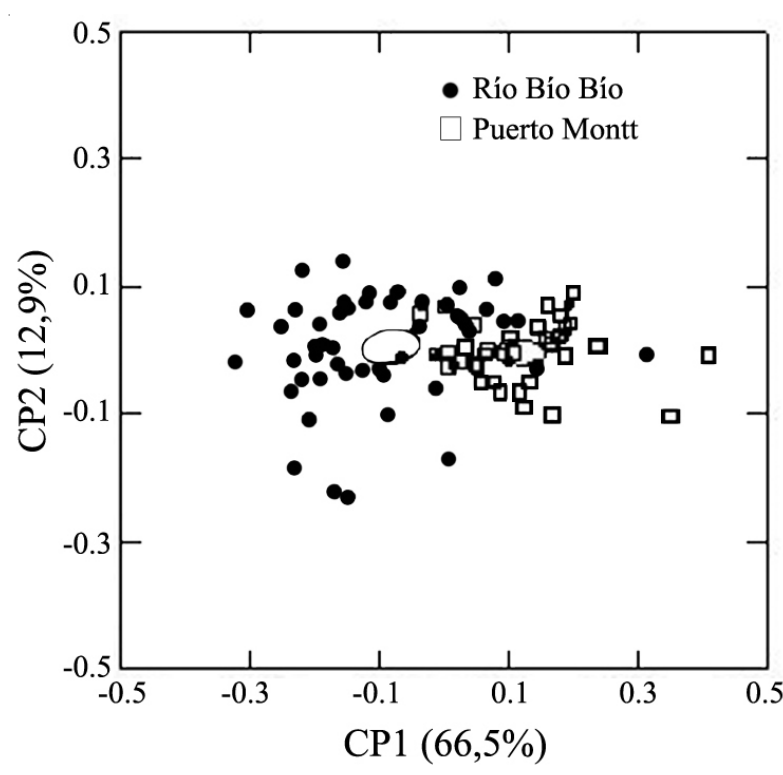

Figura 4

Ordenación de los puntajes, para las dos primeras componentes principales de un ACP realizado sobre 17 variables morfométricas de $\boldsymbol{E}$. maclovinus. Entre paréntesis porcentaje de la varianza explicada por componente $(\mathrm{CPi}=\mathrm{i}$-ésima componente principal; centroide al $95 \%$ )

ACP scores for two first principal components from the analysis of 17 morphometric variables of E. maclovinus. In brackets percentage of explained variance for each component is shown $(\mathrm{CPi}=\mathrm{i}$-th principal component; $95 \%$ centroid)

\section{Tabla 2}

ANCOVA realizado sobre los puntajes de los dos primeros ejes del ACP, utilizando como fuente de variación la localidad y como covariable la longitud total. (*: indica valores significativos; $\mathbf{L T}=$ Longitud total)

ANCOVA for the ACP scores of the two first axes, using the locality as variation source and total length as covariate $(*$ : significant values; LT $=$ Total length)

\begin{tabular}{lccccc}
\hline (CP1) & & & & & \\
\hline Fuente & Suma de cuad. & g.l. & Cuad. medios & $\mathbf{F}$ & $\mathbf{p}$ \\
\hline Localidad & 0,111 & 1 & 0,111 & 12,285 & $0,001^{*}$ \\
LT & 0,456 & 1 & 0,456 & 50,422 & $0,000^{*}$ \\
& & & & & \\
Error & 0,797 & 88 & 0,009 & & \\
\hline & & & & & \\
\hline (CP2) & & & & & \\
\hline Fuente & Suma de cuad. & g.l. & Cuad. medios & $\mathbf{F}$ & $\mathbf{p}$ \\
\hline Localidad & 0,001 & 1 & 0,001 & 0,194 & 0,661 \\
LT & 0,020 & 1 & 0,020 & 4,320 & $0,041^{*}$ \\
Error & 0,397 & 88 & 0,005 & & \\
\hline
\end{tabular}

Tabla 3

Frecuencia numérica y en porcentaje (en paréntesis) de reclasificación, resultado del ADM realizado para $E$. maclovinus; 'grupo' corresponde a la asignación por localidades; 'predicho' corresponde a la reclasificación del ADM

Numeric and percentage (in brackets) reclassification frequency, as result of Multiple Discriminant Analysis

(MDA) on E. maclovinus variables, 'grupo'

corresponds to the locality assignation, and 'predicho' corresponds to the MDA reclassification

\begin{tabular}{lrrr}
\hline \multirow{2}{*}{ GRUPO = Localidad } & \multicolumn{2}{c}{ PREDICHO } & TOTAL LOCALIDAD \\
& Río Bío Bío & Puerto Montt & \\
\hline Río Bío Bío & $51(96,2)$ & $2(3,8)$ & $\mathbf{5 3}$ \\
Puerto Montt & $2(5,3)$ & $36(94,7)$ & $\mathbf{3 8}$ \\
\hline TOTAL PREDICHO & $\mathbf{5 3}(\mathbf{5 8 , 2 )}$ & $\mathbf{3 8}(\mathbf{4 1 , 8 )}$ & $\mathbf{9 1}(\mathbf{9 6})$ \\
\hline
\end{tabular}

los puntajes de las dos primeras componentes del ACP, utilizando como fuente de variación la localidad y como covariable la longitud total, indicó que, para la CP1 existen diferencias significativas entre las localidades $(P<0,005)$, pero que dichas diferencias no son independientes del tamaño de los ejemplares $(P<0,001)$. Para CP2 en cambio, la separación por localidad no fue significativa $(P>0,6)$, observándose que también se encuentra influenciada por la talla de los peces $(P<0,05)$.

El ADM estableció diferencias significativas entre Puerto Montt y el río Bío Bío $(P<0,001)$. La matriz de reclasificación entregó sólo un $4 \%$ de mezcla entre las localidades y una reclasificación levemente mayor para los individuos del río Bío Bío (Tabla 3).

$\mathrm{Al}$ analizar los peces de tamaños similares se observó del ACP, que CP1 y CP2 explicaron el 70\% de la varianza total, presentando CP1 $(51,9 \%)$ y CP2 $(18 \%)$ el mayor poder de discriminación entre las dos localidades estudiadas, covariando nuevamente todas las medidas con la CP1 excepto la variable 8-9 (Tabla 4, Fig. 5). La prueba multivariada, resultado del ADM, mostró diferencias significativas en la morfometría de los ejemplares provenientes de las distintas localidades $(P<0,001)$. La reclasificación total (Tabla 5) fue de un $95 \%$, existiendo un porcentaje de mezcla de sólo un 5\% entre localidades.

En la Tabla 6 se pueden observar los registros de los ocho recuentos merísticos para cada una de las localidades. Además, el resultado de una prueba $t$ para la comparación de medias (Tabla 6), mostró que para ninguno de los ocho recuentos existieron diferencias significativas entre las localidades $(P>0,1)$.

El resultado del ACP realizado sobre las 8 variables merísticas consideradas (Tabla 7) mostró que CP1 y CP2 


\section{Tabla 4}

Componentes de carga de las distancias morfométricas, para el grupo de tallas similares, con las tres primeras componentes principales en $E$. maclovinus, para la variación por localidad. (*= mayor varianza explicada por variable para cada componente; $\mathbf{C P i}=\mathbf{i}$-ésima componente principal; V. E.= varianza explicada)

Component loadings of morphometric variables, for similar length group, with three first principal components for $E$. maclovinus $(*=$ highest variance per variable for each component; $\mathrm{CP}=\mathrm{i}$-th principal component; V.E.= explained variance)

\begin{tabular}{lcrr}
\hline \multicolumn{1}{c}{ Trazo } & CP1 & CP2 & CP3 \\
\hline $1-2$ & $0,014^{*}$ & 0,003 & $-0,003$ \\
$1-12$ & $0,024^{*}$ & $-0,002$ & $-0,009$ \\
$2-3$ & $0,029^{*}$ & 0,008 & $-0,006$ \\
$2-12$ & $0,027^{*}$ & 0,005 & $-0,012$ \\
$3-10$ & $0,032^{*}$ & 0,004 & 0,007 \\
$3-11$ & $0,025^{*}$ & 0,011 & 0,015 \\
$5-6$ & $0,029^{*}$ & $-0,004$ & 0,001 \\
$5-9$ & $0,031^{*}$ & 0,001 & 0,004 \\
$5-10$ & $0,033^{*}$ & 0,009 & 0,006 \\
$6-9$ & $0,023^{*}$ & 0,004 & 0,007 \\
$6-10$ & $0,028^{*}$ & 0,001 & $-0,010$ \\
$7-8$ & 0,019 & $-0,008$ & $-0,009$ \\
$7-9$ & $0,032^{*}$ & $-0,008$ & $-0,012$ \\
$8-9$ & 0,016 & $-0,062 *$ & 0,005 \\
$10-12$ & $0,031^{*}$ & 0,002 & 0,013 \\
$11-12$ & $0,033^{*}$ & 0,005 & 0,008 \\
$12-12 b$ & $0,022^{*}$ & 0,004 & $-0,011$ \\
& & & \\
V.E. (\%) & 51,884 & 17,965 & 5,720 \\
V.E. (\% acum.) & 51,884 & 69,849 & 75,569 \\
\hline
\end{tabular}

dan cuenta del 59,5\% de la varianza total, siendo CP1 con sólo un $31,8 \%$ de la varianza, la componente que explicaría la mayor diferenciación entre localidades. Por otro lado, los resultados del ADM realizado sobre los 8 recuentos merísticos utilizando como fuente de variación la localidad de muestreo, no reveló diferencias significativas entre los ejemplares obtenidos de las dos localidades $(P>0,9)$. Las frecuencias de reclasificación para la merística (Tabla 8), indican que sólo el 57\% de los individuos están bien clasificados, correspondiendo a Puerto Montt la reclasificación mayor (63\%).

\section{Discusión}

El conocimiento sobre el robalo, y en general, sobre las especies explotadas por los pescadores artesanales en Chile es limitado y se ha restringido principalmente a especies de importancia económica. En el caso particular del robalo se han realizado varios trabajos sobre la

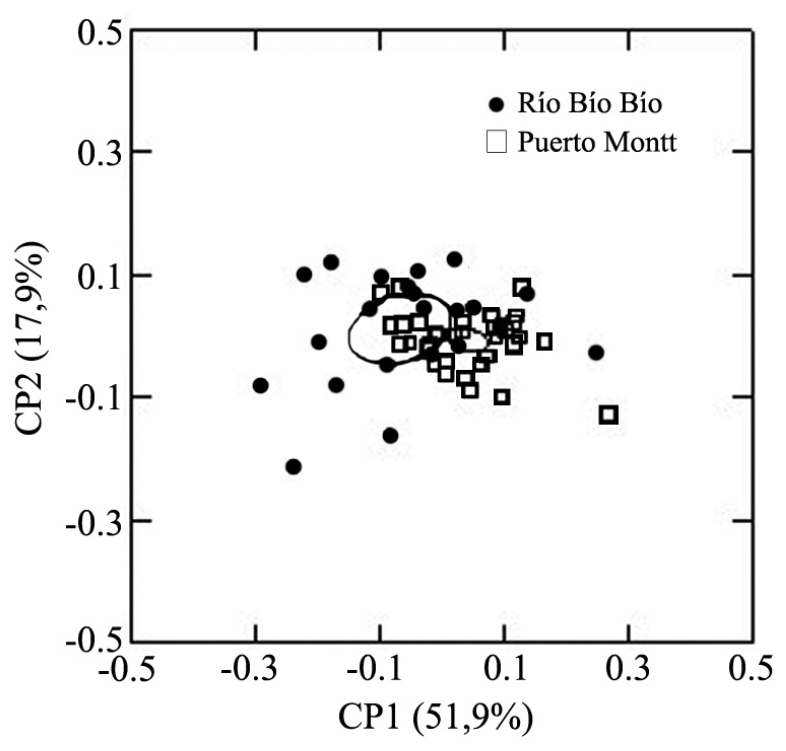

Figura 5

Ordenación de los puntajes, para las dos primeras componentes principales de un ACP realizado sobre 17 variables morfométricas de $E$. maclovinus, en grupos de peces de tamaños similares. Entre paréntesis porcentaje de la varianza explicada por componente $(\mathrm{CPi}=\mathrm{i}$-ésima componente principal; centroide al $95 \%$ )

ACP scores for the two first principal components computed from the analysis of 17 morphometric variables of $E$. maclovinus, for groups of fishes with similar length size. In brackets percentage of explained variance for each component ( $\mathrm{CPi}=\mathrm{i}$-th principal component; $95 \%$ centroid)

\section{Tabla 5}

Frecuencia numérica y en porcentaje (en paréntesis) de reclasificación por localidades, resultado del ADM realizado para el grupo de talla similares en $E$. maclovinus; 'grupo' corresponde a la asignación por localidad; 'predicho' corresponde a la reclasificación del ADM

Numeric and percentage (in brackets) reclassification frequency, as result of MDA for similar length groups of

E. Maclovinus; 'grupo' corresponds to the locality assignation, and 'predicho' corresponds to the MDA reclassification

\begin{tabular}{lrrr}
\hline \multirow{2}{*}{ GRUPO $=$ Localidad } & \multicolumn{2}{c}{ PREDICHO } & TOTAL LOCALIDAD \\
& Río Bío Bío & Puerto Montt & \\
\hline Río Bío Bío & $19(95,0)$ & $1(5,0)$ & $\mathbf{2 0}$ \\
Puerto Montt & $2(5,4)$ & $35(94,6)$ & $\mathbf{3 7}$ \\
\hline TOTAL PREDICHO & $\mathbf{2 1 ( 3 6 , 8 )}$ & $\mathbf{3 6}(\mathbf{6 3 , 2 )}$ & $\mathbf{5 7}(\mathbf{9 5})$ \\
\hline
\end{tabular}


Tabla 6

Resumen para los recuentos merísticos de 96 ejemplares de $E$. maclovinus y resultado del test $t$ realizado para la comparación de medias entre los ejemplares provenientes de la desembocadura del río Bío Bío y Puerto Montt

Summary for meristic counts of 96 E. maclovinus, and results of $t$ test mean comparison between samples from Bío Bío river and Puerto Montt

\begin{tabular}{|c|c|c|c|c|c|c|c|c|}
\hline \multirow[b]{2}{*}{ Recuento } & \multicolumn{3}{|c|}{ Río Bío Bío } & \multicolumn{3}{|c|}{ Puerto Montt } & \multirow[t]{2}{*}{$\mathbf{t}$} & \multirow[t]{2}{*}{$\boldsymbol{P}$} \\
\hline & Rango & C.V. $(\%)$ & Promedio & Rango & C.V. $(\%)$ & Promedio & & \\
\hline A. Dorsal I & $6-9$ & 8 & 7,78 & $6-9$ & 8 & 7,74 & 0,300 & 0,765 \\
\hline A. Dorsal II & $23-26$ & 3 & 25,05 & $24-26$ & 3 & 24,97 & 0,471 & 0,638 \\
\hline A. Anal & $21-25$ & 3 & 23,31 & $21-24$ & 3 & 23,21 & 0,669 & 0,505 \\
\hline A. Pectoral & $22-24$ & 2 & 22,90 & $22-25$ & 3 & 22,84 & 0,436 & 0,664 \\
\hline Branquiespinas superiores & $4-10$ & 12 & 8,12 & $7-9$ & 7 & 8,39 & $-1,177$ & 0,242 \\
\hline Branquiespinas inferiores & $13-19$ & 8 & 15,74 & $13-18$ & 8 & 15,76 & 0,329 & 0,743 \\
\hline Vértebras precaudales & $20-21$ & 2 & 20,31 & $19-22$ & 4 & 20,21 & 0,847 & 0,399 \\
\hline Vértebras caudales & $24-26$ & 2 & 24,81 & $23-27$ & 3 & 24,84 & $-0,246$ & 0,807 \\
\hline
\end{tabular}

\section{Tabla 7}

Componentes de carga para los 8 recuentos merísticos, con las 3 primeras componentes principales en $E$. maclovinus, para la separación por localidad (*= mayor varianza explicada por variable para cada componente; $\mathrm{CP}$ i=i-ésima componente principal; V.E.= varianza explicada)

Component loadings of 8 meristic counts, for the 3 first principal components computed to the locality variation in E. maclovinus

$(*=$ highest variance per variable for each component; $\mathrm{CPi}=\mathrm{i}$-th principal component; V.E.= explained variance)

\begin{tabular}{lccc}
\hline \multicolumn{1}{c}{ Recuento } & CP1 & CP2 & CP3 \\
\hline A. Dorsal I & 0,003 & 0,012 & $0,107^{*}$ \\
A. Dorsal II & 0,006 & $-0,011$ & $0,022^{*}$ \\
A. Anal & $0,013^{*}$ & 0,006 & $-0,011$ \\
A. Pectoral & 0,003 & 0,005 & $-0,026^{*}$ \\
Branquiespinas sup. & 0,087 & $0,129^{*}$ & $-0,009$ \\
Branquiespinas inf. & $0,139^{*}$ & $-0,081$ & 0,005 \\
Vértebras sup. & $0,010^{*}$ & 0,001 & $-0,008$ \\
Vértebras inf. & $0,010^{*}$ & 0,000 & 0,002 \\
& & & \\
V.E. (\%) & 31,846 & 27,644 & 15,061 \\
V.E. (\% acum.) & 31,846 & 59,490 & 74,551 \\
\hline
\end{tabular}

biología básica de la especie (Miranda 1967, Guzmán \& Campodónico 1973, Pequeño 1979, 1989, Gómez 1996, Panozo 1996, Valenzuela et al. 1999, Pavés et al. 2005), correlación entre importe de agua dulce y sus desembarques (Quiñones \& Montes 2001), como bioindicadores de contaminación en la zona centro sur de Chile (Focardi et al. 2000, Gavilán et al. 2001), sobre su edad, tasas de crecimiento y alimentación (Licandeo et al. 2006) y sobre el cariotipo (Mazzei et al. 2008). Pero existe una carencia desde el punto de vista poblacional, ya que muy poco se conoce del robalo a lo largo de su distribución geográfica, por lo que el presente
Tabla 8

Frecuencia numérica y en porcentaje (en paréntesis) de reclasificación por localidades resultado de ADM realizado sobre las 8 variables merísticas transformadas para E. maclovinus; 'grupo' corresponde a la asignación por localidades y 'predicho' a la reclasificación del ADM

Numeric and percentage (in brackets) reclassification frequency, as result of MDA on 8 transformed meristic variables for E. maclovinus; 'grupo' corresponds to the locality assignment, and 'predicho' the MDA reclassification

\begin{tabular}{lccc}
\hline \multirow{2}{*}{ GRUPO $=$ Localidad } & \multicolumn{2}{c}{ PREDICHO } & TOTAL LOCALIDAD \\
& Río Bío Bío & Puerto Montt & \\
\hline Río Bío Bío & $31(53,4)$ & $27(46,6)$ & $\mathbf{5 8}$ \\
Puerto Montt & $14(36,8)$ & $24(63,2)$ & $\mathbf{3 8}$ \\
\hline TOTAL PREDICHO & $\mathbf{4 5 ( 4 6 , 9 )}$ & $\mathbf{5 1 ( 5 3 , 1 )}$ & $\mathbf{9 6 ( 5 7 )}$ \\
\hline
\end{tabular}

trabajo, junto con el realizado por Gómez (1996), son los primeros intentos de analizar la estructura poblacional de la especie.

Los resultados del análisis de los caracteres morfométricos del robalo sugieren que existirían dos grupos discretos entre los individuos provenientes del área de Puerto Montt y de la desembocadura del río Bío Bío (Fig. 4), debe considerarse que el ANCOVA evidencia un efecto significativo de la localidad para CP1, pero que dichas diferencias se encuentran fuertemente influenciadas por la talla de los ejemplares (Tabla 2). Además, en el caso de la segunda componente, las diferencias entre las localidades no son significativas, pero el efecto de la talla de los peces se mantiene (Tabla 2). Además, del ACP se observó que casi todas las variables 
covariaron mayormente, y con signo positivo, con la primera componente principal (Tabla 1), excepto la medida 8-9 que lo hace con la CP2, y que corresponde al trazo ventral del pedúnculo caudal (Fig. 3).

De los resultados del análisis sobre el grupo del mismo rango de tallas, se obtiene del ACP y ADM conclusiones muy similares a las encontradas para el total de los datos explicando las dos primeras componentes (CP1 y CP2) casi el 77\% de la separación entre localidades (Tabla 4); siendo en este caso mucho menos clara la separación de las localidades (Fig. 5).

Humphries et al. (1981) advierten que la talla, como una variable que podría eventualmente evidenciar la influencia del tamaño sobre la forma de los peces, afectaría los resultados, conduciendo a encontrar diferencias entre los grupos analizados que pueden deberse sólo al efecto del tamaño sobre los descriptores de la forma, aun cuando estadísticamente esas diferencias sean significativas. Ello se observa más claramente al analizar los peces dentro de un mismo rango de tallas, donde las diferencias son menos claras y la mezcla entre los grupos es mayor, pero manteniéndose un grado de separación importante (Fig. 5). Por lo tanto, el efecto talla, más que influir negativamente en el análisis de las formas de los peces, puede en algunos casos aumentar la magnitud de las diferencias. Parece recomendable establecer como parte de la rutina de análisis, la comparación de grupos que se encuentre dentro de un rango similar de tallas.

Respecto de los caracteres merísticos (Tabla 6), los rangos encontrados están dentro de lo informado por la literatura (Fischer 1963, Duarte et al. 1971, Guzmán \& Campodónico 1973, Navarro \& Pequeño 1979). Al compararlos entre localidades (ACP y ADM) muestran que no existen diferencias significativas de acuerdo a la prueba multivariada realizada sobre las variables $(P>0,9)$, además, la matriz de reclasificación muestra en promedio un $43 \%$ de mezcla entre los individuos provenientes de ambas localidades (Tabla 8).

La homogeneidad encontrada para los recuentos merísticos, puede deberse a una menor plasticidad de la merística a las posibles variaciones ambientales que se presentarían entre las localidades de estudio. Esta carencia de variabilidad merística también puede estar determinada por el establecimiento de los caracteres merísticos (número de estructuras), el que se produce en los primeros estadios de desarrollo de los peces antes que éstos lleguen al estado de juveniles (Epperly 1989). Los resultados merísticos coincidirían con aquellos informados por Gómez (1996), quién establece que la variabilidad genética para muestras provenientes del río Bío Bío y Calbuco es muy baja existiendo un sólo locus polimórfico de 19 loci analizados, concluyendo que E. maclovinus sería una especie muy poco variable genéticamente.

Lo informado por Gómez (1996) concuerda con otros autores (Balushkin 1992, Klingenberg \& Ekau 1996, Lecointre et al. 1997, Bargelloni et al. 2000a, b, Eastman 2000, Eastman \& McCune 2000), en que E. maclovinus constituiría una rama ancestral durante la evolución de los Notothenioidei, el cual como grupo está caracterizado por una baja variabilidad genética (McDonald et al. 1992). Debe agregarse la condición hermafrodita del robalo (Calvo et al. 1992, Panozo 1996, Brickle et al. 2005), lo que coincidiría con la hipótesis de Balushkin (1992) que E. maclovinus sería la especie más plesiomórfica dentro del Suborden Notothenioidei (Eastman \& McCune 2000), debido a que el hermafroditismo protándrico como estrategia reproductiva es considerado un remanente evolutivo anterior, cuando las especies eran hermafroditas (Sadovy \& Shapiro 1987). Además, Valenzuela et al. (1999), en un estudio de la hematología del robalo, establecieron que la familia Eleginopsidae (Balushkin 1992) se encontraría separada de Nototheniidae y que está más relacionada con el grupo original de nototenioideos de aguas temperadas.

Finalmente, los resultados de la morfometría del robalo, a diferencia de aquellos obtenidos de la merística, permiten establecer que existirían diferencias morfológicas significativas entre los individuos de la desembocadura del río Bío Bío y aquellos provenientes del área de Puerto Montt, aun cuando se advierte un efecto del tamaño de los peces, pero dicho efecto afecta los resultados acentuando la separación entre las localidades analizadas. Siendo al parecer, una diferenciación clinal la explicación más apropiada para entender la separación encontrada, ya que se analizó poblaciones separadas por más de 700 kilómetros.

\section{Agradecimientos}

Los autores desean agradecer al M.Sc. Luis Cubillos S. y a dos revisores anónimos por los comentarios aportados para mejorar el presente manuscrito. Durante la preparación de este manuscrito R. Veas fue financiado por una beca doctoral CONICYT.

\section{Literatura citada}

Bargelloni L, L Zane, N Derome, G Lecointre \& T Patarnello. 2000a. Molecular zoogeography of Antarctic euphausiids and notothenioids: from species phylogenies to intraspecific patterns of genetic variation. Antarctic Science 12(3): 259268.

Bargelloni L, S Marcato, L Zane \& T Patarnello. 2000b. 
Mitochondrial phylogeny of notothenioids: a molecular approach to Antarctic fish evolution and biogeography. Systematic Biology 49(1): 114-129.

Balushkin A. 1992. Classification, phylogenetic relationships, and origins of the families of the Suborder Notothenioidei (Perciformes). Journal of Ichthyology 7: 90-110.

Bookstein F, B Chernoff, R Elder, J Humphries, G Smith \& R Strauss. 1985. Morphometrics in evolutionary biology. Academy of Natural Sciences of Philadelphia. Special Publication 15: 1-277.

Brickle P, V Laptikhovsky \& AArkhipkin. 2005. Reproductive strategy of a primitive temperate notothenioid Eleginops maclovinus. Journal of Fish Biology 66: 1044-1059.

Cadrin S. 2000. Advances in morphometric identification of fishery stocks. Reviews in Fish Biology and Fisheries 10(1): 91-112.

Calvo J, E Morriconi, A Rae \& NA San Roman. 1992. Evidence of protandry in subantarctic notothenid, Eleginops maclovinus (Cuv. \& Val., 1830) from the Beagle Channel, Argentina. Journal of Fish Biology 40: 157-164.

Cortés N, C Oyarzún \& R Galleguillos. 1996. Diferenciación poblacional en sardina común, Strangomera bentincki (Norman, 1936). II: Análisis multivariado de la morfometría y merística. Revista de Biología Marina 31(2): 91-105.

Duarte W, R Feito, C Jara, C Moreno \& A Orellana. 1971. Ictiofauna del sistema hidrográfico del río Maipo. Boletín del Museo Nacional de Historia Natural 32: 227-268.

Eastman JT. 2000. Antarctic notothenioid fishes as subjects for research in evolutionary biology. Antarctic Science 12(3): 276287.

Eastman JT \& AR McCune. 2000. Fishes on the Antarctic continental shelf: evolution of a marine species flock? Journal of Fish Biology 57 (Supplement A): 84-102.

Eastman JT \& RR Eakin. 2000. An updated species list for notothenioid fish (Perciformes; Notothenioidei), with comments on Antarctic species. Archives of Fishery and Marine Research 48(1): 11-20.

Epperly S. 1989. A meristic, morphometric and biochemical investigation of Atlantic menhaden Brevoortia tyranus (Latrobe). Journal of Fish Biology 26: 139-152.

Fischer WK. 1963. Die fische des Brackwassergebietes Lenga Bei, Concepcion (Chile). International Revue Gessamten Hidrobiologie 46: 419-511.

Fitzgerald DG, JW Nanson, TN Todd \& BM Davis. 2002. Application of truss analysis for the quantification of changes in fish condition. Journal of Aquatic Ecosystem Stress and Recovery 9: 115-125.

Focardi S, JC Sánchez-Hernández, R Barra, JF Gavilán \& 0 Parra. 2000. Fish Cytochrome P4501A1 activity induced by Biobio river sediments, south central Chile. Bulletin of Environmental Contamination and Toxicology 65(2): 175-182.

Gavilán JF, R Barra, MC Fossi, S Casini, G Salinas, O Parra \& S Focardi. 2001. Biochemical biomarkers in fish from different river systems reflect exposure to a variety of anthropogenic stressors. Bulletin of Environmental
Contamination and Toxicology 66(4): 476-483.

George-Nascimento M \& H Arancibia. 1992. Stocks ecológicos del jurel (Trachurus symmetricus murphyi Nichols) en tres zonas de pesca frente a Chile, detectados mediante comparación de su fauna parasitaria y morfometría. Revista Chilena de Historia Natural 65: 453-470.

Gómez D. 1996. Variabilidad genética en el robalo Eleginops maclovinus (Valenciennes, 1830) (Pisces: Perciformes: Eleginopsidae). Seminario de título de Biología Marina, Universidad de Concepción, Concepción, 40 pp.

Guzmán L \& I Campodónico. 1973. Algunos aspectos de la biología de Eleginops maclovinus (Cuv. \& Val., 1830), con especial referencia a su morfometría, caracteres merísticos y alimentación. Anales del Instituto de la Patagonia 4(1-3): 343371.

Hernández A, A Sepúlveda \& L Miranda. 1998. Morfometría y merística del jurel (Trachurus symmetricus murphyi) a lo largo de Chile. En: Arcos D (ed). Biología y Ecología del Jurel en Aguas Chilenas, pp. 47-63. Instituto de Investigación Pesquera, Talcahuano.

Humphries JM, F Bookstein, B Chernoff, G Smith, R Elder \& S Ross. 1981. Multivariate discrimination by shape in relation to size. Systematic Zoology 30(3): 291-308.

Iwami T. 2004. Comparative morphology of the adductor mandibulae musculature of notothenioid fishes (Pisces, Perciformes). Antarctic Science 16(1): 17-21.

Klingenberg CP \& W Ekau. 1996. A combined morphometric and phylogenetic analysis of an ecomorphological trend: pelagization in Antarctic fishes (Perciformes: Nototheniidae). Biological Journal of the Linnean Society 59: 141-177.

Lecointre G, C Bonillo, C Ozouf-Costaz \& JC Hureau. 1997. Molecular evidence for the origins of Antarctic fishes: paraphyly of the Bovichtidae and no indication for the monophyly of the Notothenioidei (Teleostei). Polar Biology 18: 193-208.

Licandeo R, R Barrientos \& M González. 2006. Age, growth rates, sex change and feeding habits of notothenioid fish Eleginops maclovinus from the central-southern Chilean coast. Environmental Biology of Fishes 77(1): 51-61.

Martin JP \& R Bastida. 2008. Contribución de las comunidades bentónicas en la dieta del róbalo (Eleginops maclovinus) en la ría Deseado (Santa Cruz, Argentina). Latin American Journal of Aquatic Research 36(1): 1-13.

Mazzei F, L Ghigliotti, J-P Coutanceau, HW Detrich III, V Prirodina, C Ozouf-Costaz \& E Pisano. 2008. Chromosomal characteristics of the temperate notothenioid fish Eleginops maclovinus (Cuvier). Polar Biology 31(5): 629-634.

McDonald MA, MH Smith, MW Smith, JM Novak, PE Johns \& AL DeVries. 1992. Biochemicals systematics of notothenioid fishes from Antarctica. Biochemical Systematic and Ecology 20:233-241.

Miranda O. 1967. Calendario ictiológico de San Antonio. 1. Enumeración de la comunidad de peces, mediante la red de tres telas, en un hábitat rocoso. Biología Pesquera 2: 3-49. 
Misra RK \& MDL Easton. 1999. A note on the number of morphometric characters used in fish stock delineation studies employing a MANOVA. Fisheries Research 42: 191-194.

Navarro J \& G Pequeño. 1979. Peces litorales de los archipiélagos de Chiloé y los Chonos, Chile. Revista de Biología Marina 16(3): 255-309.

Near T. 2004. Estimating divergence times of notothenioid fishes using a fossil-calibrated molecular clock. Antarctic Science 16(1): 37-44.

Oyarzún C. 1997. Análisis de la diferenciación poblacional: el caso de Merluccius gayi (Guichenot, 1848) de la costa de Chile. Tesis de Doctorado en Ciencias con Mención en Zoología, Universidad de Concepción, Concepción, 93 pp.

Pavés H, G Pequeño, C Bertrán \& L Vargas. 2005. Limnetic feeding in Eleginops maclovinus (Valenciennes, 1830) in the Valdivia River, Chile. Interciencia 30(3): 20-25.

Panozo R. 1996. Evidencias de hermafroditismo secuencial en el robalo Eleginops maclovinus Valenciennes, 1830 (Perciformes: Eleginopsidae) Seminario de Título de Biología Marina, Universidad de Concepción, Concepción, 44 pp.

Pequeño G. 1979. Antecedentes alimentarios de Eleginops maclovinus (Valenciennes, 1830) (Teleostomi Nototheniidae), en Mehuín, Chile. Acta Zoológica Lilloana 35(1): 207-230.

Pequeño G. 1987. Observations sur l'ichtyofaune des champs d'algues à Gracilaria verrucosa (Hudson) Papenfuss, 1950 de l'embouchure des rivières Quempillén et Pudeto (Chili). Cahiers de Biologie Marine 28: 361-365.

Pequeño G. 1989. The geographical distribution and taxonomic arrangement of south american nototheniid fishes (Osteichthyies, Nototheniidae). Boletín de la Sociedad de Biología de Concepción 60: 183-200.

Pequeño G, J Lamilla, D Lloris \& J Rucabado. 1995. Comparación entre las ictiofaunas intermareales de los extremos austral y boreal de los canales patagónicos. Revista de Biología Marina 30(2): 155-177.

Quiñones RA \& RM Montes. 2001. Relationship between freshwater input to the coastal zone and the historical landings of the benthic/demersal fish Eleginops maclovinus in centralsouth Chile. Fisheries Oceanography 10(4): 311-328.
Sadovy Y \& DY Shapiro. 1987. Criteria for the diagnosis of hermaphroditism in fishes. Copeia 1987(1): 136-156.

SAG. 1976. Anuario Estadístico de Pesca 1974-1975, 68 pp. División de Protección Pesquera, Ministerio de Agricultura, Chile.

Shapiro SS, MB Wilk \& HJ Chen. 1968. A comparative study of various tests for normality. Journal of American Statistic Association 63: 1343-1372.

Sokal RR \& FJ Rohlf. 1979. Biometría. Principios y métodos estadísticos en la investigación biológica, 832 pp. H Blume, Madrid.

SPSS Inc. 1998. SYSTAT Version 8.0. for Windows.[cd-rom]

StatSoft Inc. 1998. STATISTICA Version 5.1 for Windows ' 98 Edition. Tulsa.[cd-rom]

Strauss RE \& FL Bookstein. 1982. The Truss: body form reconstruction in morphometrics. Systematic Zoology 31: 113135.

Torres J, C Silva \& M Lucero. 2007. El rol de la pesca en la intensificación de las ocupaciones costeras durante el Holoceno Medio-Tardío (Bahía de Concepción, Región del Bío-Bío, Chile). Magallania 35(1): 71-93.

Valenzuela A, V Silva \& C Oyarzún. 1999. Caracterización cualitativa y cuantitativa de células sanguíneas de robalo Eleginops maclovinus (Valenciennes, 1830) (Pisces, Eleginopsidae) en la desembocadura del río Biobio, Revista de Biología Marina y Oceanografía 34(2): 261-267.

Winans G. 1987. Using morphometric and meristic characters for identifying stocks of fish. National Marine Fisheries Service Northwest and Alaska Fisheries Center. NOAA Technical Memorandum NMT S-SEFC 199: 25-62.

Zama A \& E Cárdenas. 1984a. Descriptive catalogue of marine and freshwater fishes from the Aysén Region, southern Chile, with zoogeographical notes on the fish fauna. Introduction into Aysén Chile of Pacific Salmon 9: 1-75.

Zama A \& E Cárdenas. 1984b. Predation of fishes on young salmon released into Ensenada baja, Puerto Chacabuco (XI Región, Chile). Introduction into Aysén Chile of Pacific Salmon 8: 1-11. 